Research Article

\title{
Computationally Efficient Ambiguity-Free Two-Dimensional DOA Estimation Method for Coprime Planar Array: RD-Root-MUSIC Algorithm
}

\author{
Luo Chen, ${ }^{1,2,3}$ Changbo Ye $\mathbb{D}^{1,2}$ and Baobao Li ${ }^{1,2}$ \\ ${ }^{1}$ Key Laboratory of Dynamic Cognitive System of Electromagnetic Spectrum Space (Nanjing University of Aeronautics and \\ Astronautics), Ministry of Industry and Information Technology, Nanjing 211106, China \\ ${ }^{2}$ College of Electronic and Information Engineering, Nanjing University of Aeronautics and Astronautics, Nanjing 211106, China \\ ${ }^{3}$ The $28^{\text {th }}$ Research Institute of China Electronics Technology Group Corporation, Nanjing 210007, China
}

Correspondence should be addressed to Changbo Ye; ycb@nuaa.edu.cn

Received 28 April 2020; Revised 31 May 2020; Accepted 18 June 2020; Published 10 July 2020

Guest Editor: Junpeng Shi

Copyright (c) 2020 Luo Chen et al. This is an open access article distributed under the Creative Commons Attribution License, which permits unrestricted use, distribution, and reproduction in any medium, provided the original work is properly cited.

While the two-dimensional (2D) spectral peak search suffers from expensive computational burden in direction of arrival (DOA) estimation, we propose a reduced-dimensional root-MUSIC (RD-Root-MUSIC) algorithm for 2D DOA estimation with coprime planar array (CPA), which is computationally efficient and ambiguity-free. Different from the conventional 2D DOA estimation algorithms based on subarray decomposition, we exploit the received data of the two subarrays jointly by mapping CPA to the full array of the CPA (FCPA), which contributes to the enhanced degrees of freedom (DOFs) and improved estimation performance. In addition, due to the ambiguity-free characteristic of the FCPA, the extra ambiguity elimination operation can be avoided. Furthermore, we convert the $2 \mathrm{D}$ spectral search process into $1 \mathrm{D}$ polynomial rooting via reduced-dimension transformation, which substantially reduces the computational complexity while preserving the estimation accuracy. Finally, numerical simulations demonstrate the superiority of the proposed algorithm.

\section{Introduction}

Two-dimensional direction of arrival (2D DOA) estimation has been extensively utilized in radar, sonar, wireless communication, and other fields [1-3]. Numerous DOA estimation algorithms, e.g., multiple signal classification (MUSIC) [4], estimation of signal parameters via rotational invariance techniques (ESPRIT) [5-7], propagator method (PM) [8], and PARAllel FACtor (PARAFAC) technique, have been applied to various planar arrays, such as uniform planar arrays (UPAs) [9-11], L-shaped arrays [12, 13], uniform circular arrays [5], and two parallel linear arrays [14]. However, the distance between adjacent elements in these traditional arrays is limited to no larger than halfwavelength to avoid spatial aliasing, which bring undesired serious mutual coupling effect. Besides, since the DOA estimation accuracy has positive correlation with the array aperture, the limited interelement spacing brings a negative impact on the estimation performance $[15,16]$.

In recent years, sparse arrays such as coprime arrays $[15,17,18]$, nested arrays [19], and minimum redundancy arrays [20] have been proposed to tackle this issue. As a typical sparse array, the coprime arrays have inherent superiorities over the conventional compact arrays, including enlarged array aperture, increased DOFs, and reduced mutual coupling [21], employed the traditional 2D-MUSIC algorithm to CPA by exploring the transformation relation between true and ambiguous estimates, and further proposed a 2D partial spectrum search (2D-PSS) method [22], which considerably relieves the computational burden of $2 \mathrm{D}$ total spectrum search (TSS). By combining the reduceddimensional MUSIC (RD-MUSIC) [23] method with the PSS method, the reduced-dimension transformation is performed to further reduce complexity [24]. A generalized 
CPA array structure was designed in [25] based on the mechanism of ambiguity elimination method, which provides a more flexible array layout and significant increase in DOFs. The aforementioned methods [22, 24, 25] can be categorized as decomposition algorithms, which process the received data of each subarray separately and then the estimates are combined to determine the final DOAs, whereas the mutual information between the two subarrays is unfortunately neglected. The root-MUSIC method to CPA with low complexity is applied [26], while the estimation performance of this cascade approach depends heavily on the initial estimates, especially at low SNRs. An ambiguity-free MUSIC (AF-MUSIC) method was proposed in [27], where the output of two subarrays were stacked and processed jointly to avoid the ambiguous problem. Although the entire information of CPA is fully exploited, the 2D spectrum search leads to heavy computational burden.

In the above research studies for 2D DOA estimation methods with CPA, they either treat the two subarrays as individual arrays, which suffers performance degradation due to the loss of mutual information, or $2 \mathrm{D}$ spectrum search is required leading to expensive computational cost, or extra ambiguity elimination process is involved. To address these issues, we propose a computationally efficient ambiguity-free algorithm via reduced-dimensional polynomial rooting technique. Specifically, we first map the CPA into full array of the CPA (FCPA) using an extraction matrix, based on the characteristics of array configuration, which enables the sufficient utilization of the entire received data of the CPA. Meanwhile, the number of achievable DOFs is enhanced benefiting from the utilization of full information, as compared to the conventional decomposition algorithms. Furthermore, we transform the $2 \mathrm{D}$ spectrum search into $2 \mathrm{D}$ polynomial root-finding process and further perform reduced-dimension transformation to convert the $2 \mathrm{D}$ root-finding operation into two $1 \mathrm{D}$ one, which substantially reduces complementation complexity as well as the computational burden. In addition, extra ambiguity elimination can be avoided owing to the inherent ambiguity-free characteristic of the FCPA. below:

We summarize the major contributions of our work

(1) We construct the FCPA corresponding to CPA, which processes ambiguity-free characteristic and thereby extra ambiguity elimination operation can be avoided

(2) We exploit the received data of the two subarrays jointly, where improved DOA estimation performance as well as enhanced achievable DOFs can be achieved

(3) We propose a reduced-dimensional polynomial root-finding algorithm with CPA for 2D DOA estimation, which transforms the $3 \mathrm{D}$ spectrum search into $1 \mathrm{D}$ polynomial rooting and hence reduces the complexity significantly while preserving the estimation accuracy
We outline this paper as follows. Section 2 introduces the data model of CPA and its corresponding FCPA. The proposed algorithm is elaborated in Section 3, and we analyze the complexity and DOFs in Section 4 . Section 5 provides simulation results to corroborate the effectiveness of the proposed algorithm, and Section 6 concludes this paper.

1.1. Notations. Bold uppercase (lowercase) characters represent matrices (vectors). $(\cdot)^{T},(\cdot)^{H},(\cdot)^{-1}$, and $(\cdot)^{*}$ denote the transpose, conjugate transpose, inverse, and conjugate operation, respectively. $\otimes$ and $\odot$ are Kronecker product and Khatri-Rao product, respectively. Rank $(\cdot)$ means the rank of the matrix. angle $(\cdot)$ represents the phase operator. $\operatorname{det}(\cdot)$ denotes the determinant of the matrix.

\section{Preliminaries}

2.1. Data Model with CPA. Assume that $K$ far-field narrowband uncorrelated signals impinge on the CPA with DOAs $\left(\theta_{k}, \phi_{k}\right), k=1,2, \ldots, K$, where $\theta_{k}$ and $\phi_{k}$ are the elevation and azimuth angles of the $k$ th signal, respectively. The CPA consists of two uniform planar arrays with $M_{1} \times M_{1}$ and $M_{2} \times$ $M_{2}$ sensors. The spacing between adjacent elements of subarray 1 with $M_{1} \times M_{1}$ sensors is $d_{1}=M_{2} \lambda / 2$, and subarray 2 with $M_{2} \times M_{2}$ sensors has the interelement spacing $d_{2}=M_{1} \lambda / 2$, where $M_{1}$ and $M_{2}$ are coprime integers and $\lambda$ is the wavelength. The total number of elements is $T=M_{1}^{2}+M_{2}^{2}-1$ since the two subarrays share the same element at the origin. Define a transformation as $u_{k}=\sin \theta_{k} \sin \phi_{k}$ and $v_{k}=\sin \theta_{k} \cos \phi_{k}$ for simplification. A CPA configuration is displayed in Figure 1 as an example, where $M_{1}=2, M_{2}=3$, and $T=12$.

For the $i$ th $(i=1,2)$ subarray, the received signal can be expressed by [22]

$$
\mathbf{X}_{i}=\mathbf{A}_{i} \mathbf{S}+\mathbf{N}_{i}
$$

where $\mathbf{S}$ represents the source matrix and $\mathbf{S}=\left[\mathbf{s}_{1}, \mathbf{s}_{2}, \ldots, \mathbf{s}_{K}\right]^{T} \in \mathbb{C}^{K \times L}, \mathbf{s}_{k}$ denotes source vector and $\mathbf{s}_{k}=\left[s_{k}(1), \ldots, s_{k}(L)\right]^{T} \in \mathbb{C}^{L \times 1}, L$ is the number of snapshots, $\mathbb{C}$ represents a complex set, $\mathbf{A}_{i}$ is the steering matrix of the $i$ th $(i=1,2)$ subarray, and $\mathbf{A}_{i}=\left[\mathbf{a}_{y i}\left(u_{1}\right) \otimes \mathbf{a}_{x i}\left(v_{1}\right), \ldots\right.$, $\left.\mathbf{a}_{y i}\left(u_{K}\right) \otimes \mathbf{a}_{x i}\left(v_{K}\right)\right] \in \mathbb{C}^{M_{i}^{2} \times K}, \mathbf{a}_{y i}\left(u_{k}\right)$ and $\mathbf{a}_{x i}\left(v_{k}\right)$ represent the steering vectors along the $y$-axis and $x$-axis, respectively, the specific forms can be expressed as $\mathbf{a}_{y i}\left(u_{k}\right)=\left[1, e^{j 2 \pi d_{i} u_{k} / \lambda}, \ldots, e^{j 2 \pi\left(M_{i}-1\right) d_{i} u_{k} / \lambda}\right]^{T} \in \mathbb{C}^{M_{i} \times 1} \quad$ and $\mathbf{a}_{x i}\left(v_{k}\right)=\left[1, e^{j 2 \pi d_{i} v_{k} / \lambda}, \ldots, e^{j 2 \pi\left(M_{i}-1\right) d_{i} v_{k} / \lambda}\right]^{T} \in \mathbb{C}^{M_{i} \times 1}, \quad \mathbf{N}_{i}$ is white Gaussian noise with mean value zero and variance $\sigma^{2}$ of the $i$ th $(i=1,2)$ subarray.

The output of the whole CPA can be stacked as [27]

$$
\mathbf{X}=\left[\begin{array}{l}
\mathbf{X}_{1} \\
\mathbf{X}_{2}
\end{array}\right]=\left[\begin{array}{l}
\mathbf{A}_{1} \\
\mathbf{A}_{2}
\end{array}\right] \mathbf{S}+\left[\begin{array}{l}
\mathbf{N}_{1} \\
\mathbf{N}_{2}
\end{array}\right]=\mathbf{A S}+\mathbf{N}
$$

where $\mathbf{A}$ represents the direction matrix of the whole CPA, $\mathbf{A}=\left[\mathbf{A}_{1}^{T}, \mathbf{A}_{2}^{T}\right]^{T} \in \mathbb{C}^{\left(M_{1}^{2}+M_{2}^{2}\right) \times K}$, and $\mathbf{N}$ denotes the white Gaussian noise of the whole arrays and $\mathbf{N}=\left[\mathbf{N}_{1}^{T}, \mathbf{N}_{2}^{T}\right]^{T}, \mathbf{N}_{1} \in \mathbb{C}^{M_{1}^{2} \times L}, \mathbf{N}_{2} \in \mathbb{C}^{M_{2}^{2} \times L}$. 


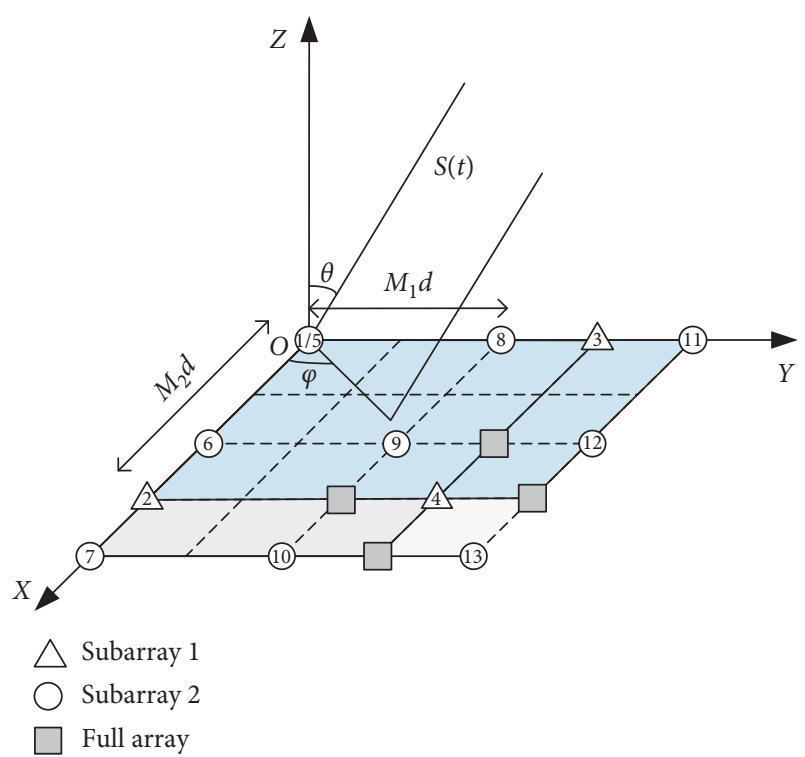

Figure 1: CPA configuration with $M_{1}=2$ and $M_{2}=3$.

In practice, the covariance matrix of $\mathbf{X}$ can be calculated using $L$ snapshots by

$$
\widehat{R}=\frac{1}{L} \mathbf{X X}^{H} .
$$

Perform eigenvalue decomposition (EVD) and $\widehat{R}$ can be decomposed by

$$
\widehat{R}=\mathbf{E}_{s} \boldsymbol{\Lambda}_{s} \mathbf{E}_{s}^{H}+\mathbf{E}_{n} \boldsymbol{\Lambda}_{n} \mathbf{E}_{n}^{H},
$$

where $\Lambda_{s}$ is a diagonal matrix whose diagonal elements are the $K$ largest eigenvalues, $\Lambda_{n}$ is a diagonal matrix composed of the rest eigenvalues, $\mathbf{E}_{s}$ represents the signal subspace spanned of the eigenvectors corresponding to the $K$ largest eigenvalues, and $\mathbf{E}_{n}$ is the noise subspace composed of the remaining eigenvectors.

2.2. Full Array of CPA and Extraction. The CPA can be extracted from a large nonuniform planar array, which can be denoted by the full array of CPA (FCPA). The sensor location of the FCPA can be expressed as

$$
\Omega_{\mathrm{FCPA}}=\left\{\Omega_{\mathrm{xFCPA}}, \Omega_{\mathrm{yFCPA}}\right\},
$$

where $\Omega_{\mathrm{xFCPA}}$ and $\Omega_{\mathrm{yFCPA}}$ represent the location sets of $x$ axis and $y$-axis, respectively. $\Omega_{\mathrm{xFCPA}}=m_{1} d_{1} \cup m_{2} d_{2}$, $\Omega_{\mathrm{yFCPA}}=m_{1} d_{1} \cup m_{2} d_{2}, \quad 0 \leq m_{1} \leq M_{1}-1, \quad 0 \leq m_{2} \leq M_{2}-1$, and $m_{1}, m_{2} \in \mathbb{Z}$. Accordingly, the number of elements in FCPA is $T_{F}=\left(M_{1}+M_{2}-1\right)^{2}$.

Figure 2 illustrates the FCPA corresponding to the CPA shown in Figure 1, where $\Omega_{\mathrm{xFCPA}}=\{0,2,3,4\} d$ and $\Omega_{\mathrm{yFCPA}}=\{0,2,3,4\} d, d=\lambda / 2$. It can be observed that the FCPA contains all elements of the CPA and has four additional elements with sensor number $7,10,12$, and 13, respectively, which demonstrates that the $\mathrm{CPA}$ can be regarded as an extraction from FCPA.

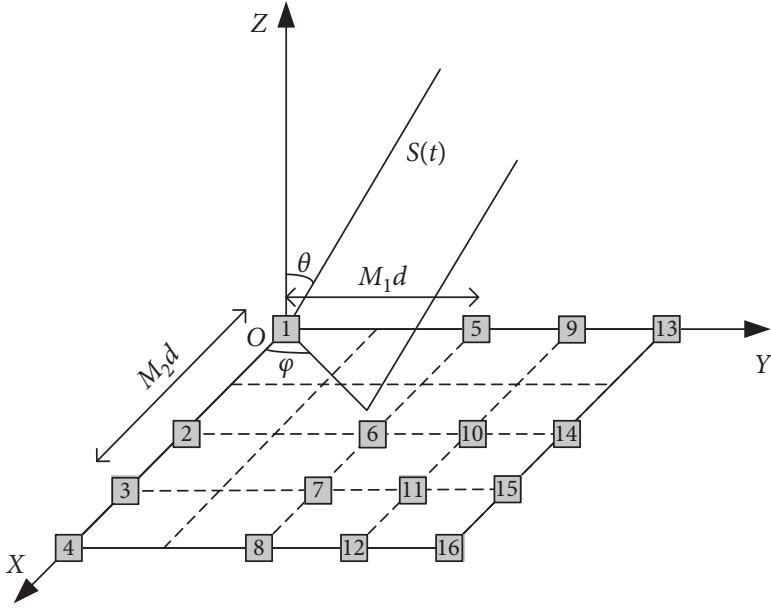

Figure 2: The FCPA configuration corresponding to CPA.

According to the correspondence of the CPA and FCPA, we introduce an extraction matrix $\mathbf{G} \in\{0,1\}$ to characterize the mapping relation as

$$
\mathbf{A}=\mathbf{G A}_{F},
$$

where $\mathbf{G} \in \mathbb{Z}^{\left(M_{1}^{2}+M_{2}^{2}\right) \times\left(M_{1}+M_{2}-1\right)^{2}}$ represents the extraction matrix, $\mathbb{Z}$ denotes a set of integers, $\mathbf{A}_{F}$ is the steering matrix of the FCPA, $\quad \mathbf{A}_{F}=\left[\mathbf{a}_{F y}\left(u_{1}\right) \otimes \mathbf{a}_{F x}\right.$ $\left.\left(v_{1}\right), \ldots, \mathbf{a}_{F y}\left(u_{K}\right) \otimes \mathbf{a}_{F x}\left(v_{K}\right)\right] \in \mathbb{C}^{\left(M_{1}+M_{2}-1\right)^{2} \times K}, \mathbf{a}_{F y}\left(u_{k}\right)$ and $\mathbf{a}_{F x}\left(v_{k}\right)$ represent the steering vector of the FCPA along the $y$-axis and $x$-axis, the specific forms are $\mathbf{a}_{F y}\left(u_{k}\right)=\left[1, e^{j 2 \pi d_{F y 2} u_{k} / \lambda}, \ldots, e^{j 2 \pi d_{F y}\left(M_{1}+M_{2}-1\right) u_{k} / \lambda}\right]^{T}$ $a_{F x}\left(v_{k}\right)=\left[1, e^{j 2 \pi d_{F x} v_{k} / \lambda}, \ldots, e^{j 2 \pi d_{F x}\left(M_{1}+M_{2}-1\right) v_{k} / \lambda}\right]^{T}$ respectively, and $d_{F y i} \in \Omega_{\mathrm{yFCPA}}, d_{F x i} \in \Omega_{\mathrm{xFCPA}}\left(1 \leq i \leq M_{1}+M_{2}-1\right)$ denote the location sets of elements on the $y$-axis and $x$-axis, respectively.

To demonstrate the extraction more specifically, each element in the CPA and FCPA is labeled according to their order in the steering vectors, i.e., $1 \sim M_{1}^{2}$ for the subarray 1 and $M_{1}^{2}+1 \sim M_{1}^{2}+M_{2}^{2}$ for the subarray 2 in CPA, $1 \sim\left(M_{1}+M_{2}-1\right)^{2}$ for the FCPA. If the $i$ th sensor in the CPA and the jth sensor in the FCPA overlap; then, $g_{i j}=1$, otherwise $g_{i j}=0$, where $g_{i j}$ denotes the $(i, j)$ th element of $\mathbf{G}$. For the FCPA given in Figure 2, G is a $13 \times 16$ matrix with 3 columns of all zeros.

Definition 1 (extraction efficiency). The extraction efficiency is the proportion of nonzero elements in the extraction matrix.

The sensor location of CPA can be given by

$$
\Omega_{\mathrm{CPA}}=\left\{\Omega_{\mathrm{xCPA}}, 0\right\} \cup\left\{0, \Omega_{\mathrm{yCPA}}\right\},
$$

where $\quad \Omega_{\mathrm{xCPA}}=m_{1} d \cup m_{2} d_{2}, \quad \Omega_{\mathrm{yCPA}}=m_{1} d_{1} \cup m_{2} d_{2}$, $0 \leq m_{1} \leq M_{1}-1,0 \leq m_{2} \leq M_{2}-1$, and $m_{1}, m_{2} \in \mathbb{Z}$. Then, we construct a uniform planar array that has the same array aperture as the CPA with the location set:

$$
\Omega_{\mathrm{UPA}}=\left\{\Omega_{\mathrm{xUPA}}, 0\right\} \cup\left\{0, \Omega_{\mathrm{yUPA}}\right\},
$$


where $\quad \Omega_{\mathrm{xUPA}}=m d, \quad \Omega_{\mathrm{yUPA}}=m d, \quad d=\lambda / 2$, $0 \leq m \leq M_{1}\left(M_{2}-1\right)$, and $m \in \mathbb{Z}$.

The locations of the sensors in the FCPA corresponding to the CPA can be expressed as

$$
\Omega_{\mathrm{FCPA}}=\left\{\Omega_{\mathrm{xFCPA}}, \Omega_{\mathrm{yFCPA}}\right\},
$$

where $\Omega_{\mathrm{xCPA}} \subseteq \Omega_{\mathrm{xFCPA}} \subseteq \Omega_{\mathrm{xUPA}}$ and $\Omega_{\mathrm{yCPA}} \subseteq \Omega_{\mathrm{yFCPA}} \subseteq \Omega_{\mathrm{yUPA}}$ represent the location set of FCPA on the $x$ - and $y$-axes, respectively.

For the CPA study in this paper, the corresponding FCPA can be constructed by the following four forms:

$$
\begin{aligned}
\Omega_{\mathrm{xFCPA}} & =\{0,2,3,4\} d, \\
\Omega_{\mathrm{yFCPA}} & =\{0,2,3,4\} d, \\
\Omega_{\mathrm{xFCPA} \_1} & =\{0,2,3,4\} d, \\
\Omega_{\mathrm{yFCPA} \_1} & =\{0,1,2,3,4\} d, \\
\Omega_{\mathrm{xFCPA} \_2} & =\{0,1,2,3,4\} d, \\
\Omega_{\mathrm{yFCPA} \_2} & =\{0,2,3,4\} d, \\
\Omega_{\mathrm{xFCPA} \_3} & =\{0,1,2,3,4\} d, \\
\Omega_{\mathrm{yFCPA} \_3} & =\{0,1,2,3,4\} d .
\end{aligned}
$$

According to Definition 1, the extraction efficiency of the above schemes is $0.0625,0.05,0.05$, and 0.04 , respectively. It is clearly seen that the FCPA we designed in (5) has the highest extraction efficiency.

\section{The Proposed Algorithm}

3.1. 2D-MUSIC Algorithm. Derive from the orthogonal relationship between the noise subspace and the steering vector, and the spectral function of CPA can be represented by $[26,27]$

$$
P(u, v)=\frac{1}{\mathbf{a}^{H}(u, v) \mathbf{E}_{n} \mathbf{E}_{n}^{H} \mathbf{a}(u, v)},
$$

where $\mathbf{a}(u, v)$ denotes the steering vector of CPA, $\mathbf{a}(u, v)=\left[\mathbf{a}_{1}^{T}(u, v), \mathbf{a}_{2}^{T}(u, v)\right]^{T} \in \mathbb{C}^{\left(M_{1}^{2}+M_{2}^{2}\right) \times 1}, \quad \mathbf{a}_{i}(u, v)=$ $\mathbf{a}_{y i}(u) \otimes \mathbf{a}_{x i}(v), \quad \mathbf{a}_{y i}(u)=\left[1, e^{j 2 \pi d_{i} u / \lambda}, \ldots, e^{j 2 \pi\left(M_{i}-1\right) d_{i} u / \lambda}\right]^{T}$, $a_{x i}(v)=\left[1, e^{j 2 \pi d_{i} v / \lambda}, \ldots, e^{j 2 \pi\left(M_{i}-1\right) d_{i} v / \lambda}\right]^{T}(i=1,2)$, and $\mathbf{E}_{n}$ is the noise subspace of CPA.

According to (6), we have $\mathbf{a}(u, v)=\mathbf{G a}_{F}(u, v)$. Then, (11) can be rewritten as $[26,27]$

$$
P(u, v)=\frac{1}{\mathbf{a}_{F}^{H}(u, v) \mathbf{G}^{H} \mathbf{E}_{n} \mathbf{E}_{n}^{H} \mathbf{G a}_{F}(u, v)}=\frac{1}{\mathbf{a}_{F}^{H}(u, v) \mathbf{E}_{F n} \mathbf{E}_{F n}^{H} \mathbf{a}_{F}(u, v)},
$$

where $\mathbf{a}_{F}(u, v)$ is the steering vector of the FCPA, $\mathbf{a}_{F}(u, v)=\mathbf{a}_{F y}(u) \otimes \mathbf{a}_{F x}(v) \in \mathbb{C}^{\left(M_{1}+M_{2}-1\right)^{2} \times 1}, \quad \mathbf{a}_{F y}(u)=$ $\left[1, e^{j 2 \pi d_{F y 2} u / \lambda}, \ldots, e^{j 2 \pi d_{F y\left(M_{1}+M_{2}-1\right)} u / \lambda}\right]^{T} \in \mathbb{C}^{\left(M_{1}+M_{2}-1\right) \times 1}$, $\mathbf{a}_{F x}(v)=\left[1, e^{j 2 \pi d_{F x 2} v / \lambda}, \ldots\right.$,

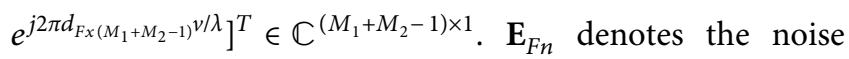
space of the FCPA, and $\mathbf{E}_{F n}=\mathbf{G}^{H} \mathbf{E}_{n}$.

Although the autopaired 2D DOA estimates can be obtained via performing spectral search on (12), it suffers from tremendously expensive computational cost. To tackle this issue, we first performed reduced-dimension transformation and then exploited 1D polynomial root-finding technique to estimate $u$ and $v$.

3.2. Reduced-Dimensional Polynomial Root-Finding Process. Construct the polynomial based on (12) as

$$
\begin{aligned}
V(u, v)= & {\left[\mathbf{a}_{F y}(u) \otimes \mathbf{a}_{F x}(v)\right]^{H} \mathbf{E}_{F n} \mathbf{E}_{F n}^{H}\left[\mathbf{E}_{F y}(u) \otimes \mathbf{a}_{F x}(v)\right] } \\
= & \mathbf{a}_{F x}^{H}(v)\left[\mathbf{a}_{F y}(u) \otimes \mathbf{I}_{M_{1}+M_{2}-1}\right]^{H} \\
& \mathbf{E}_{F n} \mathbf{E}_{F n}^{H}\left[\mathbf{a}_{F y}(u) \otimes \mathbf{I}_{M_{1}+M_{2}-1}\right] \mathbf{a}_{F x}(v) \\
= & \mathbf{a}_{F x}^{H}(v) \mathbf{Q}(u) \mathbf{a}_{F x}(v), \\
V(u, v)= & {\left[\mathbf{a}_{F y}(u) \otimes \mathbf{a}_{F x}(v)\right]^{H} \mathbf{E}_{F n} \mathbf{E}_{F n}^{H}\left[\mathbf{a}_{F y}(u) \otimes \mathbf{a}_{F x}(v)\right] } \\
= & \mathbf{a}_{F y}^{H}(u)\left[\mathbf{I}_{M_{1}+M_{2}-1} \otimes \mathbf{a}_{F x}(v)\right]^{H} \\
& \mathbf{E}_{F n} \mathbf{E}_{F n}^{H}\left[\mathbf{I}_{M_{1}+M_{2}-1} \otimes \mathbf{a}_{F x}(v)\right] \mathbf{a}_{F y}(u) \\
= & \mathbf{a}_{F y}^{H}(u) \mathbf{Q}(v) \mathbf{a}_{F y}(u),
\end{aligned}
$$

where $\quad \mathbf{Q}(u)=\left[\mathbf{a}_{F y}(u) \otimes \mathbf{I}_{M_{1}+M_{2}-1}\right]^{H} \mathbf{E}_{F n} \mathbf{E}_{F n}^{H}\left[\mathbf{a}_{F y}(u)\right.$ $\left.\otimes \mathbf{I}_{M_{1}+M_{2}-1}\right] \quad$ and $\quad \mathbf{Q}(v)=\left[\mathbf{I}_{M_{1}+M_{2}-1} \otimes \mathbf{a}_{F x}(v)\right]^{H} \mathbf{E}_{F n} \mathbf{E}_{F n}^{H}$ $\left[\mathbf{I}_{M_{1}+M_{2}-1} \otimes \mathbf{a}_{F x}(v)\right]$.

According to the relation of rank of the matrix product, the following constraint

$$
0<\operatorname{Rank}\left(\mathbf{E}_{F n} \mathbf{E}_{F n}^{H}\right) \leq \operatorname{Rank}\left(E_{F n}\right)
$$

has to be satisfied, and then we have

$$
\operatorname{det}\left\{\mathbf{E}_{F n} \mathbf{E}_{F n}^{H}\right\} \neq 0 \text {. }
$$

We can conclude that $\operatorname{det}\{\mathbf{Q}(u)\}$ is nonzero polynomial from (16); thus, $\mathbf{Q}(u)$ is a factor of $V(u, v)$. As $\mathbf{Q}(u)$ depends only on the variable $u$, the roots of $\operatorname{det}\{\mathbf{Q}(u)\}=0$ can make the following equation hold:

$$
V(u, v)=\mathbf{a}_{F x}^{H}(v) \mathbf{Q}(u) \mathbf{a}_{F x}(v)=0 .
$$

It is noteworthy that only $1 \mathrm{D}$ polynomial is involved to achieve the estimates of $u$. Similarly, we can get the estimates of $v$. Consequently, the problem of obtaining paired estimates of $u$ and $v$ from the $2 \mathrm{D}$ polynomial is transformed into two $1 \mathrm{D}$ root-finding process. Then, we reconstruct (13) and (14) as 


$$
\begin{aligned}
& \operatorname{det}\{\mathbf{Q}(u)\}=\operatorname{det}\left\{\left[\mathbf{a}_{F y}(u) \otimes \mathbf{I}_{M_{1}+M_{2}-1}\right]^{H} \mathbf{E}_{F n} \mathbf{E}_{F n}^{H}\left[\mathbf{a}_{F y}(u) \otimes \mathbf{I}_{M_{1}+M_{2}-1}\right]\right\}=0, \\
& \operatorname{det}\{\mathbf{Q}(v)\}=\operatorname{det}\left\{\left[\mathbf{I}_{M_{1}+M_{2}-1} \otimes \mathbf{a}_{F x}(v)\right]^{H} \mathbf{E}_{F n} \mathbf{E}_{F n}^{H}\left[\mathbf{I}_{M_{1}+M_{2}-1} \otimes \mathbf{a}_{F x}(v)\right]\right\}=0 .
\end{aligned}
$$

Define

$$
\begin{aligned}
& z_{1}=e^{j 2 \pi d u / \lambda} \\
& z_{2}=e^{j 2 \pi d v / \lambda}
\end{aligned}
$$

where $d=\lambda / 2$.

Define the steering vector of UPA that has the same array aperture as FCPA along the $x$-axis as $\mathbf{a}_{E x}(v)=\left[1, e^{j 2 \pi d v / \lambda}\right.$, $\ldots, e^{j 2 \pi \min }\left\{M_{1}, M_{2}\right\}\left(\max \left\{M_{1}, M_{2}\right\}-1\right)$

$d v / \lambda]^{T} \in \mathbb{C}^{\left(\left(\min \left\{M_{1}, M_{2}\right\} \max \left\{M_{1}, M_{2}\right\}-1\right)+1\right) \times 1}$. We assume that $M_{1}<M_{2}$ for simplification and then $\mathbf{a}_{E x}(v)$ can be expressed as $\mathbf{a}_{E x}(v)=\left[1, e^{j 2 \pi d v / \lambda}\right.$, $\left.\ldots, e^{j 2 \pi M_{1}\left(M_{2}-1\right) d v / \lambda}\right]^{T} \in \mathbb{C}^{\left(M_{1}\left(M_{2}-1\right)+1\right) \times 1}$. Based on the correspondence between FCPA and UPA with same array aperture, we have

$$
\mathbf{a}_{F x}(v)=\mathbf{G}_{1} \mathbf{a}_{E x}(v),
$$

where $\mathbf{G}_{1} \in \mathbb{Z}^{\left(M_{1}+M_{2}-1\right) \times\left(M_{1}\left(M_{2}-1\right)+1\right)}$. To be specific, $g_{1 i j}=1$ holds when the $i$ th sensor in the $\mathbf{a}_{F x}(v)$ and the $j$ th sensor in the $\mathbf{a}_{E x}(v)$ overlap, otherwise $g_{i j}=0$, where $g_{i j}$ is the $(i, j)$ th element of $\mathbf{G}_{1}$. For the FCPA displayed in Figure $2, \mathbf{G}_{1}$ is a $4 \times 5$ matrix with one columns of all zeros. Similarly, we can obtain the relation $\mathbf{a}_{F y}(u)=\mathbf{G}_{1} \mathbf{a}_{E y}(u)$.

Correspondingly, the steering vectors can be rewritten as

$$
\begin{aligned}
& \mathbf{a}_{F y}(u)=\mathbf{G}_{1} \mathbf{a}_{E y}(u)=\mathbf{G}_{1}\left[1, e^{j 2 \pi d u / \lambda}, \ldots, e^{j 2 \pi M_{1}\left(M_{2}-1\right) d u / \lambda}\right]^{T}=\mathbf{G}_{1}\left[1, z_{1}, \ldots, z_{1}^{M_{1}\left(M_{2}-1\right)}\right]^{T}=\infty_{F y}\left(z_{1}\right), \\
& \mathbf{a}_{F x}(v)=\mathbf{G}_{1} \mathbf{a}_{E x}(v)=\mathbf{G}_{1}\left[1, e^{j 2 \pi d v / \lambda}, \ldots, e^{j 2 \pi M_{1}\left(M_{2}-1\right) d v / \lambda}\right]^{T}=\infty_{1}\left[1, z_{2}, \ldots, z_{2}^{M_{1}\left(M_{2}-1\right)}\right]^{T}=\mathbf{a}_{F x}\left(z_{2}\right) .
\end{aligned}
$$

$\underset{z_{1}^{M_{1}\left(M_{2}-1\right)}\left[\mathbf{a}_{F y}^{T}\left(z_{1}^{-1}\right) \otimes \mathbf{I}_{M_{1}+M_{2}-1}\right]^{H} \text { for }\left[\mathbf{a}_{F y}(u) \otimes \mathbf{I}_{M_{1}+M_{2}-1}\right]^{H}}{\text { githout }}$ and substituting $z_{2}^{M_{1}\left(M_{2}-1\right)}\left[\mathbf{I}_{M_{1}+M_{2}-1} \otimes \mathbf{a}_{F x}^{T}\left(z_{2}^{-1}\right)\right]^{H}$ for
$\left[\mathbf{I}_{M_{1}+M_{2}-1} \otimes \mathbf{a}_{F x}(v)\right]^{H}$, i.e.,

$$
\begin{aligned}
& \operatorname{det}\left\{\mathbf{Q}\left(z_{1}\right)\right\}=\operatorname{det}\left\{z_{1}^{M_{1}\left(M_{2}-1\right)}\left[\mathbf{a}_{F y}^{T}\left(z_{1}^{-1}\right) \otimes \mathbf{I}_{M_{1}+M_{2}-1}\right]^{H} \mathbf{E}_{n} \mathbf{E}_{n}^{H}\left[\mathbf{a}_{F y}\left(z_{1}^{-1}\right) \otimes \mathbf{I}_{M_{1}+M_{2}-1}\right]\right\}=0, \\
& \operatorname{det}\left\{\mathbf{Q}\left(z_{2}\right)\right\}=\operatorname{det}\left\{z_{2}^{M_{1}\left(M_{2}-1\right)}\left[\mathbf{I}_{M_{1}+M_{2}-1} \otimes \mathbf{a}_{F x}^{T}\left(z_{2}^{-1}\right)\right]^{H} \mathbf{E}_{n} \mathbf{E}_{n}^{H}\left[\mathbf{I}_{M_{1}+M_{2}-1} \otimes \mathbf{a}_{F x}\left(z_{2}^{-1}\right)\right]\right\}=0 .
\end{aligned}
$$

Considering that $\operatorname{det}\left\{\mathbf{Q}\left(z_{1}\right)\right\}$ and $\operatorname{det}\left\{\mathbf{Q}\left(z_{2}\right)\right\}$ are polynomials of even degree, $\widehat{u}_{k}$ and $\widehat{v}_{i}$ can be obtained from the $K$ roots distributed closest to the unit circle corresponding to (24) and (25), and the roots are denoted by $\widehat{z}_{11}, \ldots, \widehat{z}_{1 k}, \ldots, \widehat{z}_{1 K}$ and $\widehat{z}_{21}, \ldots, \widehat{z}_{2 i}, \ldots, \widehat{z}_{2 K}$, respectively, i.e.,

$$
\begin{gathered}
\widehat{u}_{k}=\left(\frac{\operatorname{angle}\left(\widehat{z}_{1 k}\right) \lambda}{2 \pi d}\right), \quad k=1, \ldots, K, \\
\widehat{v}_{i}=\left(\frac{\operatorname{angle}\left(\widehat{z}_{2 i}\right) \lambda}{2 \pi d}\right), \quad i=1, \ldots, K .
\end{gathered}
$$

For the conventional DOA estimation methods with CPA, the ambiguity elimination operation is required since the interelement spacing in the two subarrays is larger than half-wavelength. The FCPA is an unambiguous array which has at least one sensor pair with separation no larger than half-wavelength according to (5), and we can obtain the true
DOA estimates directly after the parameter pairing without extra ambiguity elimination process.

3.3. Parameter Pairing and DOA Estimation. In this part, we determine the pairing of $\widehat{u}_{k}$ and $\widehat{v}_{i}$ since the two root-finding procedures are conducted separately. Construct the cost function for pairing as

$$
V_{k, i}=\underset{i=1, \ldots, K}{\arg \min }\left\|\mathbf{a}^{H}\left(\widehat{u}_{k}, \widehat{v}_{i}\right) \mathbf{E}_{n} \mathbf{E}_{n}^{H} \mathbf{a}\left(\widehat{u}_{k}, \widehat{v}_{i}\right)\right\|(k=1, \ldots, K),
$$

where $\mathbf{a}\left(\widehat{u}_{k}, \widehat{v}_{i}\right)$ represents the steering vector reconstructed $\widehat{u}_{k}$ and $\widehat{v}_{i}$, which can be obtained according to (1).

For each $\widehat{u}_{k}$, we can obtain the value of $i$ and $k$ that minimize $V_{k, i}(1 \leq i \leq K)$, and we define the paired index as $i^{\prime}$ and $k$. Finally, the 2D DOAs can be calculated by 


$$
\begin{aligned}
& \widehat{\theta}_{k}=\arcsin \left(\sqrt{\widehat{u}_{k}^{2}+\widehat{v}_{i}^{2}}\right), \quad 1 \leq k \leq K, \\
& \widehat{\phi}_{k}=\arctan \left(\frac{\widehat{u}_{k}}{\widehat{v}_{i^{\prime}}}\right), \quad 1 \leq k \leq K,
\end{aligned}
$$

where $\widehat{v}_{i^{\prime}}$ is reconstructed by $i^{\prime}\left(1 \leq i^{\prime} \leq K\right)$.

3.4. The Procedure of the Proposed Algorithm. We summarize the major steps of the proposed algorithm as follows:

Step1: calculate $\widehat{R}$ of the received data $\mathbf{X}$ and perform EVD to obtain the noise space $\mathbf{E}_{n}$

Step 2: reconstruct the spectral function $P(u, v)$ according to (6)

Step 3: construct the polynomial $V(u, v)$ and conduct reduced-dimension transformation according to (12)

Step 4: calculate $\widehat{u}_{k}$ and $\widehat{v}_{i}$ according to (26) and (27) Step 5: perform parameter matching to obtain $\widehat{\theta}_{k}$ and $\widehat{\phi}_{k}$ according to (28)-(30)

\section{Performance Analysis}

4.1. Complexity Analysis. Herein, we compare the computational complexity of the proposed algorithm, 2D-PSS [22], RD-MUSIC [23], AF-MUSIC [27], and 2D-ROOT [26] methods in this section. For the proposed algorithm, calculating the covariance matrix requires $O\left\{T^{2} L\right\}$ and the complexity of eigenvalue decomposition is $O\left\{T^{3}\right\}$. The rootfinding operation costs $O\left\{2\left(2 M_{1}\left(M_{2}-1\right)\right)^{3}\right\}$ and parameter matching process requires $O\left\{K^{2}(T-K)(T+1)\right\}$. Consequently, the total complexity is $O\left\{T^{2} L+T^{3}+2\left(2\left(M_{1}\left(M_{2}-1\right)\right)\right)^{3}+K^{2}(T-K)(T+1)\right\}$.

Table 1 lists the total complexity of above algorithms, where $\Delta=0.0001$ is the spectral search interval. In addition, Figure 3 displays the complexity comparison versus number of sensors, where $K=2$ and $L=500$, while the complexity comparison with different number of snapshots is shown in Figure 4 , where $K=2, M_{1}=2$, and $M_{2}=3$. It is clearly seen that the proposed algorithm owns the approximate low complexity to the 2D-ROOT method, which is significantly lower than that of the 2D-PSS, RD-MUSIC, and AF-MUSIC methods, as the spectral search process with heavy computational burden is transformed into computationally efficient polynomial root-finding.

4.2. Cramer-Rao Bound. In this part, the derivation of Cramer-Rao Bound (CRB) of the 2D DOA estimation with $\mathrm{CPA}$ is given as the performance comparison metric.

Define $\mathbf{A}=\left[\begin{array}{l}\mathbf{A}_{1} \\ \mathbf{A}_{2}\end{array}\right]$, where $\mathbf{A}_{1}=\left[\mathbf{a}_{y 1}\left(\theta_{1}, \phi_{1}\right) \otimes \mathbf{a}_{x 1}\left(\theta_{1}\right.\right.$, $\left.\left.\phi_{1}\right), \ldots, \mathbf{a}_{y 1}\left(\theta_{K}, \phi_{K}\right) \otimes \mathbf{a}_{x 1}\left(\theta_{K}, \phi_{K}\right)\right]$ and $\mathbf{A}_{2}=\left[\mathbf{a}_{y 2}\left(\theta_{1}, \phi_{1}\right)\right.$ $\left.\otimes \mathbf{a}_{x 2}\left(\theta_{1}, \phi_{1}\right), \ldots, \mathbf{a}_{y 2}\left(\theta_{K}, \phi_{K}\right) \otimes \mathbf{a}_{x 2}\left(\theta_{K}, \phi_{K}\right)\right]$. According to [28], the CRB of CPA can be given by

$$
\mathrm{CRB}=\frac{\sigma^{2}}{2 L}\left\{\operatorname{Re}\left[\mathbf{D}^{H} \boldsymbol{\Pi}_{\mathbf{A}}^{\perp} \mathbf{D} \oplus \widehat{P}^{T}\right]\right\}^{-1},
$$

where $\mathbf{D}=\left[\partial \mathbf{a}_{1} / \partial \theta_{1}, \ldots, \partial \mathbf{a}_{K} / \partial \theta_{K}, \partial \mathbf{a}_{1} / \partial \phi_{1}, \ldots, \partial \mathbf{a}_{K} / \partial \phi_{K}\right]$, $\Pi_{\mathbf{A}}^{\perp}=\mathbf{I}_{M_{1}^{2}+M_{2}^{2}-1}-\mathbf{A}\left[\mathbf{A}^{H} \mathbf{A}\right]^{-1} \mathbf{A}^{H}, \widehat{P}=\left[\begin{array}{ll}\widehat{P}_{s} & \widehat{P}_{s} \\ \widehat{P}_{s} & \widehat{P}_{s}\end{array}\right], \widehat{P}_{s}=\mathbf{S S}^{H} / L$, $\mathbf{a}_{k}$ denotes the $k$ th column of $\mathbf{A}$, and $\sigma^{2}$ is the variance of the received noise.

4.3. Achievable DOFs. As for the conventional decomposition-based 2D DOA estimation methods, such as RDMUSIC, 2D-PSS, and other algorithms, the number of achievable DOFs is $\min \left\{M_{1}^{2}-1, M_{2}^{2}-1\right\}$. According [26], $M_{1}^{2}+M_{2}^{2}-2$ signals at most can be resolved by utilizing AF-MUSIC algorithm which processes the received data of the two subarrays jointly, whereas extremely high computational complexity is involved due to the $2 \mathrm{D}$ spectral peak search. RD root-finding technique is employed in the proposed algorithm to deal with the complexity and the achievable DOFs can be obtained from (15). If $u$ or $v$ does not match any of the incident signals, we have the constraint

$$
\max \left\{M_{1}, M_{2}\right\} \leq \operatorname{Rank}\left(\mathbf{E}_{F n} \mathbf{E}_{F n}^{H}\right) \leq \operatorname{Rank}\left(\mathbf{E}_{F n}\right),
$$

where

$\operatorname{Rank}\left(\mathbf{E}_{F n}\right)=\min \left\{\left(M_{1}+M_{2}-1\right)^{2}, M_{1}^{2}+M_{2}^{2}-1-K\right\}$.

Consequently, the maximum number of signals which can be identified by the proposed algorithm is

$$
K \leq M_{1}^{2}+M_{2}^{2}-1-\max \left\{M_{1}, M_{2}\right\}
$$

It is clear that the proposed algorithm can greatly improve the DOFs compared with the traditional 2D DOA estimation methods.

4.4. Advantages. Based on the above discussion, the advantages of the proposed algorithm can be listed as follows:

(1) It can achieve superior estimation performance and higher achievable DOFs than the decomposition methods, owing to the utilization of entire received data. Moreover, additional ambiguity elimination is no longer required due to the ambiguity-free characteristic of the FCPA.

(2) It outperforms the AF-MUSIC algorithm, RDMUSIC algorithm, 2D-PSS algorithm, and 2DROOT algorithm in estimation performance.

(3) The proposed algorithm owns much lower computational complexity than the RD-MUSIC, 2D-PSS, and AF-MUSIC methods, which is approximately as low as 2D-ROOT algorithm.

\section{Simulations}

In this section, we perform 500 Monte Carlo simulations to validate the performance of the proposed algorithm. Assume 
TABLE 1: Complexity of different algorithms.

\begin{tabular}{|c|c|}
\hline Algorithm & Complexity \\
\hline Proposed & $O\left\{T^{2} L+T^{3}+2\left(2\left(M_{1}\left(M_{2}-1\right)\right)^{2}\right)^{3}+K^{2}(T-K)(T+1)\right\}$ \\
\hline 2D-PSS & $O\left\{\left(M_{1}^{4}+M_{2}^{4}\right) L+M_{1}^{6}+M_{2}^{6}+4 M_{1}^{2}\left(M_{1}^{2}-K\right) /\left(M_{2}^{2} \Delta^{2}\right)+4 M_{2}^{2}\left(M_{2}^{2}-K\right) /\left(M_{1}^{2} \Delta^{2}\right)\right\}$ \\
\hline RD-MUSIC & $O\left\{\begin{array}{c}\left(M_{1}^{4}+M_{2}^{4}\right) L+M_{1}^{6}+M_{2}^{6}+2\left(2 M_{1}^{3}\left(M_{1}^{3}-K\right)+M_{1}^{4}+M_{1}^{3}\right) / \Delta \\
+2\left(2 M_{2}^{3}\left(M_{2}^{3}-K\right)+M_{2}^{4}+M_{2}^{3} / \Delta\right)\end{array}\right.$ \\
\hline 2D-ROOT & $O\left(T^{2} L+T^{3}+8\left(M_{1}^{6}+K M_{1}^{3}+M_{2}^{6}+K M_{2}^{3}\right)\right)$ \\
\hline AF-MUSIC & $O\left(T^{2} L+T^{3}+8 T(T-K) / \Delta^{2}\right)$ \\
\hline
\end{tabular}
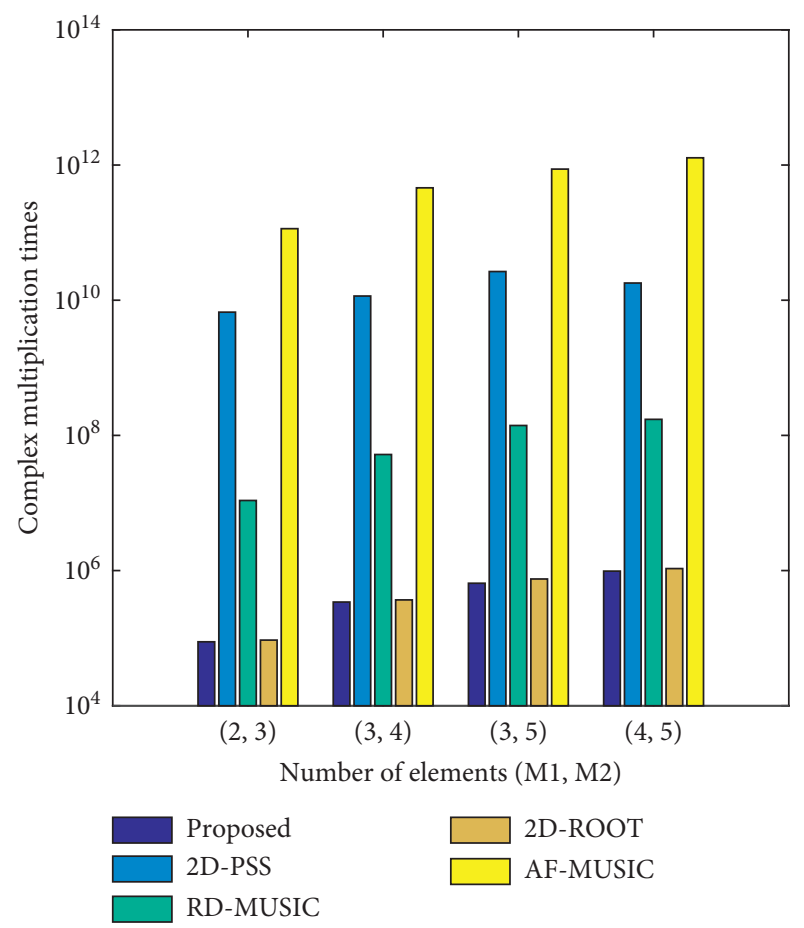

FIGURE 3: Complexity comparison versus number of elements.

that $K$ uncorrelated far-field narrowband signals impinge on the CPA. Define root mean square error (RMSE) by

$$
\mathrm{RMSE}=\frac{1}{K} \sum_{k=1}^{K} \sqrt{\frac{1}{500} \sum_{i=1}^{500}\left(\hat{\phi}_{k, i}-\phi_{k}\right)^{2}+\left(\widehat{\theta}_{k, i}-\theta_{k}\right)^{2}},
$$

where $\widehat{\phi}_{k, i}$ and $\widehat{\theta}_{k, i}$ are the estimates of the $k$ th signal in the $i$ th trial corresponding to the true azimuth $\phi_{k}$ and elevation $\theta_{k}$, respectively.

5.1. Scatter Figures. The scatter figures of the proposed algorithm under $K$ sources are as follows, where $K=6, \mathrm{SNR}=30 \mathrm{~dB}, L=1000, \quad\left(\theta_{1}, \theta_{2}, \theta_{3}, \theta_{4}, \theta_{5}, \theta_{6}\right)=\left(5^{\circ}\right.$, $\left.15^{\circ}, 25^{\circ}, 35^{\circ}, 45^{\circ}, 55^{\circ}\right)$, and $\left(\phi_{1}, \phi_{2}, \phi_{3}, \phi_{4}, \phi_{5}, \phi_{6}\right)=\left(5^{\circ}, 15^{\circ}\right.$, $\left.25^{\circ}, 35^{\circ}, 45^{\circ}, 55^{\circ}\right)$. The CPA is composed of two UPAs with $M_{1} \times M_{1}=2 \times 2$ and $M_{2} \times M_{2}=3 \times 3$. As illustrated in Figure 5 , the proposed algorithm can effectively distinguish all sources incident on the CPA.

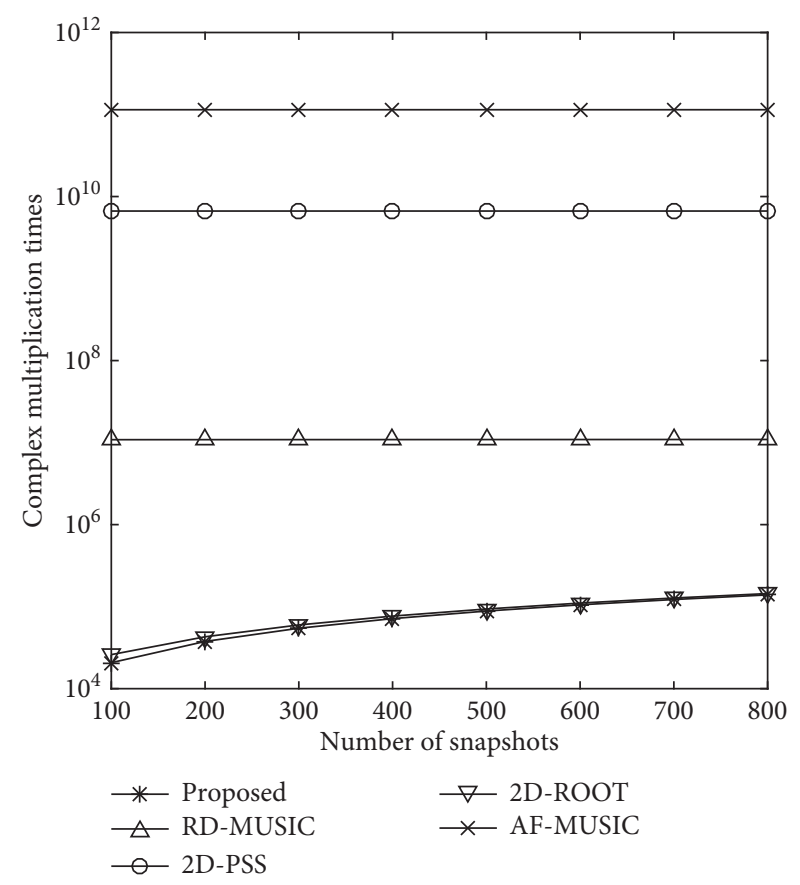

FIGURE 4: Complexity comparison versus snapshots.

5.2. RMSE Results Versus Snapshots. In this part, we present the DOA estimation performance of the proposed algorithm with different number of snapshots in Figure 6, where $\left(\theta_{1}, \phi_{1}\right)=\left(20^{\circ}, 30^{\circ}\right),\left(\theta_{2}, \phi_{2}\right)=\left(40^{\circ}, 50^{\circ}\right), M_{1} \times M_{1}=2 \times 2$, and $M_{2} \times M_{2}=3 \times 3$. The result demonstrates that the estimation accuracy improves as the number of snapshots increases, owing to the more accurate covariance.

5.3. RMSE Results Versus Number of Sensors. Herein, we provide the RMSE results of the proposed algorithm versus number of sensors in Figure 7 , where $\left(\theta_{1}, \phi_{1}\right)=\left(20^{\circ}, 30^{\circ}\right)$, $\left(\theta_{2}, \phi_{2}\right)=\left(40^{\circ}, 50^{\circ}\right)$, and $L=200$. As the number of array elements increases, the diversity gain of the receiving antenna increases. It is illustrated clearly that the increased number of sensors leads to improved DOA estimation performance.

5.4. RMSE Comparison of Different Algorithms. Figure 8 exhibits the RMSE comparison of the proposed 


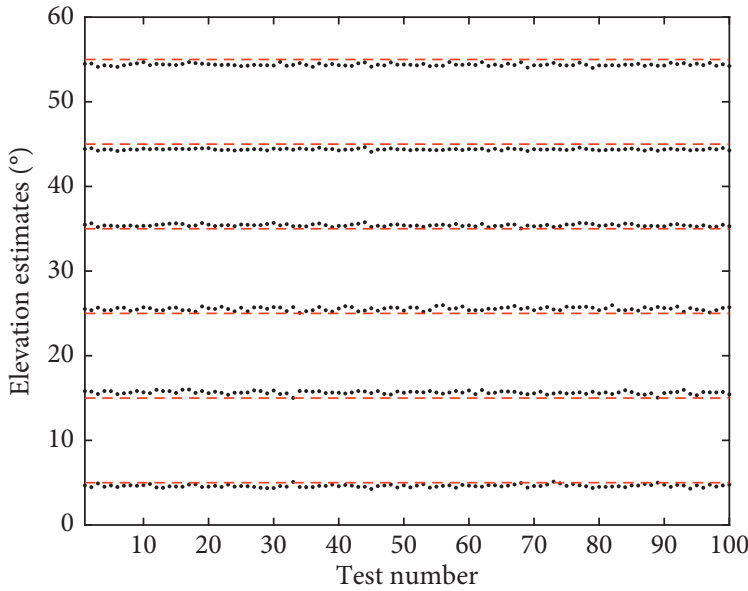

(a)

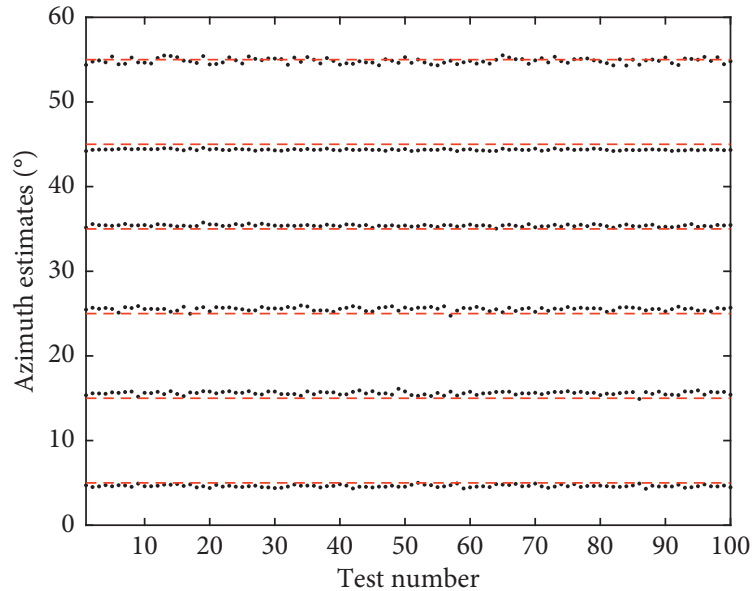

(b)

Figure 5: (a) Elevation estimates of the proposed algorithm. (b) Azimuth estimates of the proposed algorithm.

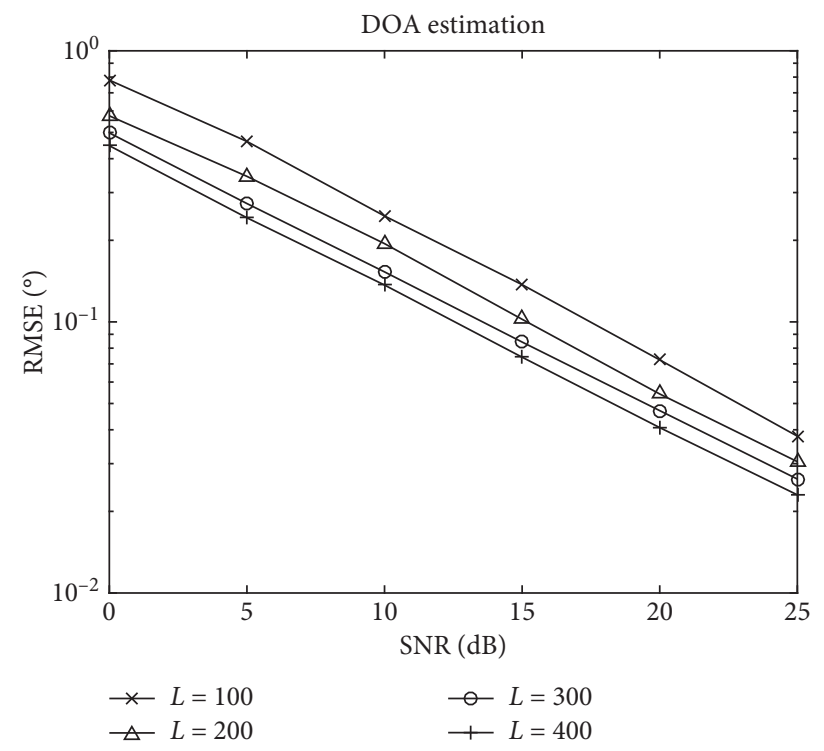

FIGURE 6: RMSE performance of the proposed algorithm versus snapshots.

algorithm, 2D-ROOT algorithm, 2D-PSS algorithm, RDMUSIC, and AF-MUSIC algorithm, where $M_{1} \times M_{1}=2 \times 2$ and $M_{2} \times M_{2}=3 \times 3$. It is indicated explicitly in Figure 8 that the proposed algorithm and AF-MUSIC algorithm, benefiting from the utilization of the received data of the entire CPA, outperform the decomposition-based RDMUSIC, 2D-PSS, and 2D-ROOT algorithms. Furthermore, the proposed algorithm yields superior estimation performance to the AF-MUSIC algorithm, as the proposed algorithm directly performs two root-finding operations based on the spectral function (11), while the cascading process in the AF-MUSIC algorithm may result in performance degradation.

\section{Conclusion}

In this paper, we propose a computationally efficient $2 \mathrm{D}$ DOA estimation algorithm for CPA by exploiting the RD polynomial root-finding technique. The proposed algorithm first maps CPA into FCPA and exploits the received data of two subarrays jointly, where the mutual information loss is avoided and simultaneously the improved estimation performance as well as enhanced DOFs can be achieved. In particular, the FCPA we constructed is an ambiguity-free array with high extraction efficiency. Furthermore, we convert the 2D total spectral search into one 1D polynomial root-finding process via reduced- 


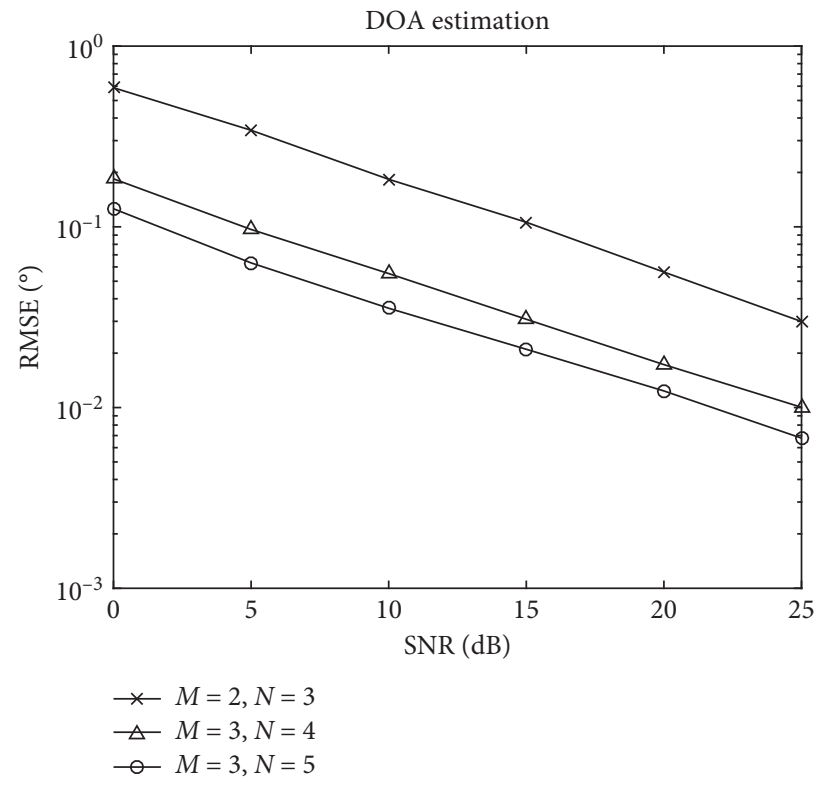

FIGURE 7: RMSE performance of the proposed algorithm versus number of sensors.

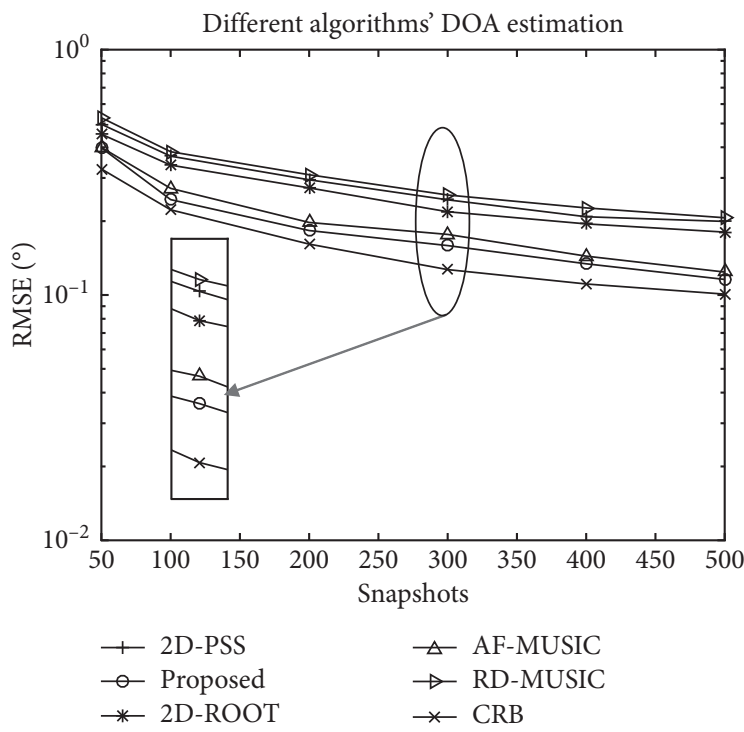

(a)

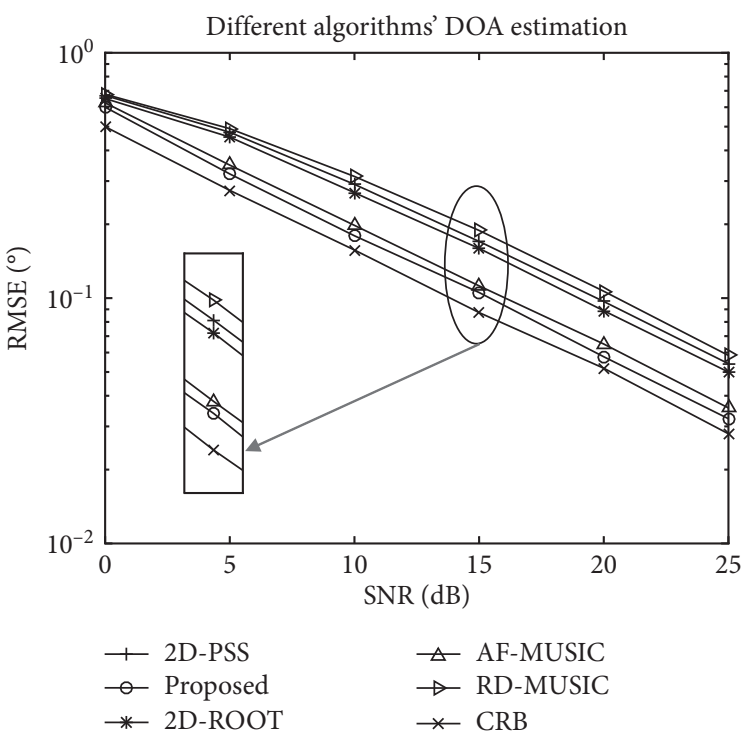

(b)

FIGURE 8: (a) RMSE comparison of different algorithms versus snapshots. (b) RMSE comparison of different algorithms versus SNR.

dimension transformation, which significantly reduces the computational cost and simultaneously preserves the estimation accuracy. Simulations demonstrates the superiority of the proposed approach in regard to complexity, achievable DOFs and DOA estimation performance.

\section{Data Availability}

The data used to support the findings of this study are available from the corresponding author upon reasonable request.

\section{Conflicts of Interest}

The authors declare that they have no conflicts of interest.

\section{Acknowledgments}

This work was supported by the China NSF (Grant nos. 61631020, 61971218, 61601167, and 61371169), Fund of Sonar Technology Key Laboratory (Research on the theory and algorithm of signal processing for two-dimensional underwater acoustics coprime array), and Fund of SONAR 
Technology Key Laboratory (Range estimation and location technology of passive target via multiple array combination).

\section{References}

[1] H. Krim and M. Viberg, "Two decades of array signal processing research: the parametric approach," IEEE Signal Processing Magazine, vol. 13, no. 4, pp. 67-94, 1996.

[2] L. C. Godara, "Applications of antenna arrays to mobile communications: performance improvement, feasibility, and system considerations," Proceedings of the IEEE, vol. 85, no. 7 , pp. 1031-1060, 1997.

[3] L. C. Godara, "Application of antenna arrays to mobile communications: beam-forming and direction-of-arrival considerations," Proceedings of the IEEE, vol. 85, no. 8, pp. 1195-1245, 1997.

[4] M. Wax, T. J. Tie-Jun Shan, and T. Kailath, "Spatio-temporal spectral analysis by eigenstructure methods," IEEE Transactions on Acoustics, Speech, and Signal Processing, vol. 32, no. 4, pp. 817-827, 1984.

[5] C. P. Mathews and M. D. Zoltowski, "Eigen-structure techniques for 2-D angle estimation with uniform circular arrays," IEEE Transactions on Signal Processing, vol. 42, no. 9, pp. 3295-3306, 1994.

[6] H. Saarnisaari, "TLS-ESPRIT in a time delay estimation," in Proceedings of IEEE Vehicular Technology Conference, pp. 1619-1623, Phoenix, AZ, USA, May 1997.

[7] G. Xu, S. D. Silverstein, R. H. Roy et al., "Beamspace esprit," IEEE Transactions on Signal Processing, vol. 42, no. 2, pp. 349-356, 1994.

[8] N. Tayem and H. M. Kwon, "L-shape 2-dimensional arrival angle estimation with propagator method," IEEE Transactions on Antennas and Propagation, vol. 53, no. 5, pp. 1622-1630, 2005.

[9] W. Zhang, W. Liu, J. Wang, and S. Wu, "Computationally efficient 2-D doa estimation for uniform rectangular arrays," Multidimensional Systems and Signal Processing, vol. 25, no. 4, pp. 847-857, 2014.

[10] Y. M. Chen, J. H. Lee, and C. C. Yeh, “Two-dimensional angleof-arrival estimation for uniform planar arrays with sensor position errors," IEE Proceedings F Radar and Signal Processing, vol. 140, no. 1, pp. 37-42, 1993.

[11] P. Heidenreich, A. M. Zoubir, and M. Rubsamen, "Joint 2-D doa estimation and phase calibration for uniform rectangular arrays," IEEE Transactions on Signal Processing, vol. 60, no. 9, pp. 4683-4693, 2012.

[12] Y. Hua, T. K. Sarkar, and D. D. Weiner, "An l-shaped array for estimating 2-d directions of wave arrival," IEEE Transactions on Antennas and Propagation, vol. 39, no. 2, pp. 143-146, 1991.

[13] S. O. Al-Jazzar, D. C. Mclernon, and M. A. Smadi, "SVDbased joint azimuth/elevation estimation with automatic pairing," Signal Processing, vol. 90, no. 5, pp. 1669-1675, 2010.

[14] H. Chen, C.-P. Hou, Q. Wang, L. Huang, and W.-Q. Yan, "Cumulants-based toeplitz matrices reconstruction method for 2-d coherent doa estimation," IEEE Sensors Journal, vol. 14, no. 8, pp. 2824-2832, 2014.

[15] P. P. Vaidyanathan and P. Pal, "Sparse sensing with co-prime samplers and arrays," IEEE Transactions on Signal Processing, vol. 59, no. 2, pp. 573-586, 2011.

[16] R. Schmidt, "Multiple emitter location and signal parameter estimation," IEEE Transactions on Antennas and Propagation, vol. 34, no. 3, pp. 276-280, 1986.
[17] P. Pal and P. P. Vaidyanathan, "Coprime sampling and the music algorithm," in Proceedings of the 2011 Digital Signal Processing and Signal Processing Education Meeting (DSP/ SPE), Sedona, AZ, USA, January 2011.

[18] S. Qin, Y. D. Zhang, and M. G. Amin, "Generalized coprime array configurations for direction-of-arrival estimation," IEEE Transactions on Signal Processing, vol. 63, no. 6, pp. 13771390, 2015.

[19] P. Pal and P. P. Vaidyanathan, "Nested arrays: a novel approach to array processing with enhanced degrees of freedom," IEEE Transactions on Signal Processing, vol. 58, no. 8, pp. 4167-4181, 2010.

[20] A. Moffet, "Minimum-redundancy linear arrays," IEEE Transactions on Antennas and Propagation, vol. 16, no. 2, pp. 172-175, 1968.

[21] J. Shi, G. Hu, X. Zhang, F. Sun, and H. Zhou, "Sparsity-based two-dimensional doa estimation for coprime array: from sum-difference coarray viewpoint," IEEE Transactions on Signal Processing, vol. 65, no. 21, pp. 5591-5604, 2017.

[22] Q. Wu, F. Sun, P. Lan, G. Ding, and X. Zhang, "Two-dimensional direction-of-arrival estimation for co-prime planar arrays: a partial spectral search approach," IEEE Sensors Journal, vol. 16, no. 14, pp. 5660-5670, 2016.

[23] X. Zhang, L. Xu, L. Xu, and D. Xu, "Direction of departure (dod) and direction of arrival (doa) estimation in mimo radar with reduced-dimension music," IEEE Communications Letters, vol. 14, no. 12, pp. 1161-1163, 2010.

[24] W. Zheng, X. Zhang, and Z. Shi, "Two-dimensional direction of arrival estimation for coprime planar arrays via a computationally efficient one-dimensional partial spectral search approach," IET Radar Sonar \& Navigation, vol. 11, no. 10, pp. 1581-1588, 2017.

[25] W. Zheng, X. Zhang, and H. Zhai, "Generalized coprime planar array geometry for 2-d doa estimation," IEEE Communications Letters, vol. 21, no. 5, pp. 1075-1078, 2017.

[26] D. Zhang, Y. Zhang, G. Zheng et al., "Two-dimensional direction of arrival estimation for coprime planar arrays via polynomial root finding technique," IEEE Access, vol. 6, 2018.

[27] W. Zheng, X. Zhang, L. Xu et al., "Unfolded coprime planar array for $2 \mathrm{~d}$ direction of arrival estimation: an apertureaugmented perspective," IEEE Access, vol. 6, pp. 22744-22753, 2018.

[28] P. Stoica and A. Nehorai, "Performance study of conditional and unconditional direction-of-arrival estimation," IEEE Transactions on Acoustics, Speech, and Signal Processing, vol. 38, no. 10, pp. 1783-1795, 1990. 